\title{
Minimum Entropy Approach For Robot Manipulator
}

Skaf, Zakwan and Al-Bayati, Ahmad and Wang, Hong 2011

MIMS EPrint: 2012.11

Manchester Institute for Mathematical Sciences

School of Mathematics

The University of Manchester

\footnotetext{
Reports available from: http://eprints.maths.manchester.ac.uk/

And by contacting: The MIMS Secretary

School of Mathematics

The University of Manchester

Manchester, M13 9PL, UK
} 


\title{
Minimum Entropy Approach For Robot Manipulator
}

\author{
Zakwan Skaf, Ahmad AI-Bayati and Hong Wang
}

\begin{abstract}
In this paper, a new algorithm for an adaptive PI controller for nonlinear systems subject to stochastic nonGaussian disturbance is studied. The minimum entropy control is applied to decrease the closed-loop tracking error on an ILC basis. The key issue here is to divide the control horizon into a number of equal time intervals called batches. Within each interval, there are a fixed number of sample points. The design procedure is divided into two main algorithms, within each batch and between any two adjacent batches. A D-type ILC law is employed to tune the PI controller coefficients between two adjacent batches. However, within each batch, the PI coefficients are fixed. A sufficient condition is established to guarantee the stability of the closed-loop system. An analysis of the ILC convergence is carried out. Two-link robot manipulator example is included to demonstrate the use of the control algorithm, and satisfactory results are obtained.
\end{abstract}

\section{INTRODUCTION}

Research into controller design for stochastic systems has been regarded as an important aspect of this field of research over the past few decades. This is mainly due to the fact that a large class of physical systems has random inputs, time delays, uncertainties and noise. Therefore, an ideal control design should be such that the system attenuates the stochastic behaviour, or sufficiently minimizes the effect caused by the randomness. In most stochastic or PDF control system designs, the output tracking error signal (the difference between the set point and the system output) of the closed-loop control system is a central index that represents the control performance of the closed-loop systems. Under the assumption that the random variables or the noise in the stochastic system are subject to Gaussian processes, the tracking error is used to represent the closed-loop performance. The statistics of the tracking error thus characterize the performance of the controller. The first approach to controller design focused on mean and variance control [1], minimizing the uncertainties of the closed-loop stochastic systems. Later on, linear optimal control [2] and linear quadratic martingale control [3] were developed. Further research has been performed into optimal stochastic control, optimal adaptive predictive control for nonlinear stochastic systems, and stochastic adaptive control strategies presented in [4], [5], [6], respectively. Also, the sliding mode control

Z. Skaf is with Control Systems Center, School of Electrical and Electronic Engineering, Sackville Street Building,University of Manchester, Manchester, M60 1QD, UK Zakwan.Skaf@manchester.ac.uk

A. AI-Bayati is with Control Systems Center, School of Electrical and Electronic Engineering, Sackville Street Building,University of Manchester, Manchester, M60 1QD, UK Ahmad.Al-Bayati@postgrad.manchester.ac.uk

H. Wang is with Control Systems Center, School of Electrical and Electronic Engineering, Sackville Street Building,University of Manchester, Manchester, M60 1QD, UK Hong.Wang@manchester.ac.uk problem for a class of linear continuous-time systems with Markovian jump parameters was solved in [7], and robust fuzzy control for uncertain Markovian stochastic systems was developed in [8]. More recently, robust $H_{\infty}$ control of uncertain stochastic, nonlinear systems [9], and an adaptive output-feedback controller for a stochastic nonlinear system [10] were introduced.

On the other hand, when the random variables or the noise in the stochastic system are subject to non-Gaussian processes (i.e., systems with non-symmetrically distributed noises), the mean and variance are insufficient to characterize the stochastic properties, since the spread of the non-symmetrically distributed noises cannot be accurately described by the variance of the tracking error measures alone [11]. This is because the shape of the tracking error PDF will be related to both the control parameters and the statistics of the noise. Therefore, a new measure of randomness, called the entropy of the tracking error of the closed-loop system, should be employed for the closed-loop control design of non-Gaussian stochastic systems. Thus, the main objective of minimum entropy control would be formulating a deterministic control signal to minimize the entropy of the closed-loop tracking error. Entropy has a significant advantage in dealing with non-Gaussian systems. This is because entropy provides a general description of the uncertainties of stochastic systems, without constraints the of using certain distributions. Moreover, it has been proved that the minimum entropy algorithm is similar to the minimum variance algorithm, when the noise of the stochastic system follows a Gaussian behaviour [12], [13]. For an arbitrary continuous random variable $x \in[a, b]$. The entropy of a $x$ is defined as

$$
H(x)=-\int_{a}^{b} \gamma(x) \ln \gamma(x) d x
$$

where $\gamma(x)$ is the PDF of $x$. From (1) it is clear that the entropy is a measure of the amount of uncertainty represented by the probability distribution and provides a more general character of the stochastic system, which is subject to arbitrary noises with any PDF shape. Suppose $x$ is a Gaussian variable, $a=-\infty$, and $b=+\infty$. Also, consider that the PDF of $x$ is Gaussian distribution defined as follows.

$$
\gamma(x)=\frac{1}{\sqrt{2 \pi} \sigma} \exp \left(-\frac{(x-m)^{2}}{2 \sigma^{2}}\right)
$$

where $m$ and $\sigma^{2}$ are the mean and the variance of the distribution, respectively. By substituting (2) in (1), it can be seen that the entropy $H(x)$ can be expressed as.

$$
H(x)=0.5\left(1+\ln \left(2 \pi \sigma^{2}\right)\right)
$$


From (3), it can be seen that minimizing entropy is equivalent to the minimization of the variance of $x$, and in this way, minimum variance control may be regarded as a special case of minimum entropy control. Entropy was used in different definitions. Initially, the entropy was presented as a measure of the uncertainty about its actual structure in both continuous and discrete time [14]. Later on, a new definition was presented in [15] by considering the exponential nature of the information gain function. Also, the entropy was represented as an average information content in a given probability density function [16]. In fact, entropy concept was applied widely in the design of control algorithms for stochastic systems. Among various existing approaches is the work of [12], where the entropy was applied to characterize and minimize the closed loop randomness for general stochastic systems subject to arbitrary bounded random inputs. The control input was formulated which minimizes the output entropy and guarantee the local stability of the closed-loop system. Later on, in [13], the authors developed a control algorithm for the control of the output mean values and the minimization of the closed-loop entropy for nonlinear affine and non-Gaussian stochastic systems. Moreover, a novel controller design for a linear time-invariant stochastic system subject to a bounded random input by using Youla parameterization was established in [17]. Also, a minimum entropy control problem was solved for nonlinear ARMA systems over a communication network with a stochastic delay in the communication channels, where the probability density function of the tracking error was estimated by using a neural network [18].

\section{PRoblem Formulation}

In this chapter, the following nonlinear stochastic model between the output and input will be considered

$$
\begin{aligned}
x(i+1) & =A x(i)+B u(i)+g(x(i))+d(i) \\
y(i) & =C x(i)
\end{aligned}
$$

where $x(i) \in R^{n}$ is the state vector, $u(i) \in R^{r}$ is the measurable input vector and $y(i) \in R^{p}$ is the measurable output vector. Moreover, $A, B$ and $C$ represent the known parametric matrices of the dynamic part of the system with suitable dimensions. $d(i)$ is the bounded non-Gaussian random noise. $g(x(i))$ is a nonlinear vector function that stands for the nonlinear dynamics of the model and is supposed to satisfy $g(0)=0$, and the following Lipschitz condition, similar to [19]

$$
\left\|g\left(x_{1}(i)\right)-g\left(x_{2}(i)\right)\right\| \leq\left\|U\left(x_{1}(i)-x_{2}(i)\right)\right\|
$$

for any $x_{1}(i)$ and $x_{2}(i)$, where $\mathrm{U}$ is a known matrix. Denoting the desired system output as $r(i)$, the tracking error can be expressed as

$$
e(i)=r(i)-y(i)
$$

which is a non-Gaussian random process due to the affect of the non-Gaussian disturbance on the closed-loop performance. Theoretically, it is expected that such a randomness or uncertainty is minimized by the controller function. In other words, the purpose of the PI controller design in this chapter is to establish a control signal so that the randomness or uncertainty in the system output and closed-loop tracking error is minimized. The controller coefficients will be trained by an ILC tuning mechanism to minimize the entropy of the closed-loop tracking error. For this purpose, the well-known Renyis entropy measure for a random error $(e)$ can be expressed as follows

$$
H(e)=\frac{1}{1-\alpha} \log \left(\int \gamma^{\alpha}(e) d e\right)
$$

where $\gamma$ presents the probability density function of the random error. The following Kernel density estimation method is used to estimate the tracking error probability distribution function within each batch using the sampled tracking error data [16].

$$
\gamma(e) \approx \hat{\gamma}(e)=\frac{1}{N} \sum_{i=1}^{N} K_{\sigma}\left(e-e_{i}\right)
$$

where $K_{\sigma}($.$) is a real, symmetrical Kernel function with the$ specifications stated in [16], and $\sigma>0$ is the order of Renyis quadratic entropy. The chosen Kernel function in this work is expressed as follows:

$$
K_{\sigma}(x)=\frac{1}{\sqrt{2 \pi}} \exp \left(\frac{-x^{2}}{2}\right)
$$

The choice of the Kernel function is actually dependent on the level of smoothness the designer expects from the pdf estimation. The ILC-based controller tuning objective function can be written as follows:

$$
\left.H(e)=\frac{1}{1-\alpha} \log \left(V_{R a}(e)\right)\right)
$$

where $V_{R a}(e)$ is usually called the Information Potential (IP), and can be further expressed as

$$
V_{R a}(e)=\frac{1}{N^{\alpha}} \sum_{i=1}^{N}\left[\sum_{j=1}^{N} K_{\sigma}\left(e_{i}-e_{j}\right)\right]^{\alpha}
$$

\section{Controller Algorithm Design}

PI controllers are widely used in both theoretical studies and practical applications of industrial control over the last five decades [20]. The popularity of PI controller can be attributed to its robust performance in a wide range of operating conditions and to its very simple structure.

As discussed before, the aim of the PI controller design is to minimize the randomness of the tracking error, so that the entropy of the tracking error can be minimized. For this purpose, the control horizon will be divided into a number of equal time-domain intervals called batches as $\left\{T^{1}, T^{2}, T^{3}, \ldots . T^{k}, \ldots.\right\}$. Within each interval, there are a fixed number of sample points $\{1,2 \ldots . ., \mathrm{N}\}$, where $N$ is sampling numbers in a batch. In this case, the batch length $N$ should be selected large enough so that the system almost reaches the steady state with each batch. In each batch, a number of 


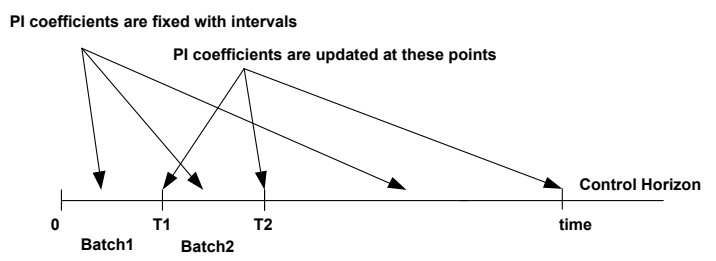

Fig. 1. PI-tuning procedure

sampled tracking error can be collected. Between any two adjacent batches, the PI coefficients are tuned by ILC-based method to minimize the tracking error entropy ( see Fig. (1)). The ILC tuning mechanism of PI coefficients needs to go on at the beginning of the $k$ batches until the PI coefficients cannot be further tuned.

A generalized PI controller with tunable coefficients is considered as adaptive controller in this work as follows

$$
\begin{aligned}
& \xi(i)=\xi(i-1)+T_{s} e(i-1) \\
& u(i)=K_{P} e(i)+K_{I} \xi(i)
\end{aligned}
$$

where $e(i)=r-y(i)$ and $T_{s}$ is the sampling time.

Assume that the current instant is at the beginning of the interval $T^{k}$, the system tracking error within this interval should have the following sample values $\left\{e_{1}^{k}, e_{2}^{k}, e_{3}^{k}, \ldots . e_{N}^{k}\right\}$. Within this interval, the sampled tracking error can be used to measure the entropy and construct the PDF. Therefore, the entropy of sample $\left\{e_{1}^{k-1}, e_{2}^{k-1}, e_{3}^{k-1}, \ldots . e_{N}^{k-1}\right\}$ in the interval $T^{k-1}$ can be expressed as follows

$H^{k-1}(e)=\frac{1}{1-\alpha} \log \left\{\frac{1}{N_{\alpha}} \sum_{i=1}^{N}\left[\sum_{i=1}^{N} K_{\sigma}\left(e_{i}^{k-1}-e_{j}^{k-1}\right]^{\alpha-1}\right\}\right.$

and the information potential for $\left\{e_{1}^{k-1}, e_{2}^{k-1}, e_{3}^{k-1}, \ldots . e_{N}^{k-1}\right\}$ in the interval $T^{k-1}$ can be expressed according to the following equation

$$
V_{R a}^{k-1}(e)=\frac{1}{N^{\alpha}} \sum_{i=1}^{N}\left[\sum_{j=1}^{N} K_{\sigma}\left(e_{i}^{k-1}-e_{j}^{k-1}\right)\right]^{\alpha-1}
$$

By substituting (14) into (13), the entropy can be obtained as follows:

$$
H^{k-1}(e)={\frac{1}{1-\alpha_{R a}}}^{k-1}(e)
$$

The task of tunning the PI controller coefficients between adjacent batches can be solved by the following D-type ILC law which can be only considered as a local optimal solution

$$
\begin{aligned}
& K_{P}(k)=K_{P}(k-1)-\lambda_{P} \frac{\partial H^{k-1}(e)}{\partial K_{P}^{k-1}} \\
& K_{I}(k)=K_{I}(k-1)-\lambda_{I} \frac{\partial H^{k-1}(e)}{\partial K_{I}^{k-1}}
\end{aligned}
$$

where $\lambda_{P}$ and $\lambda_{I}$ are the ILC learning rates chosen so that the iterative control law is convergent [21]. $K_{P}(k)$ and $K_{I}(k)$ are the PI controller coefficients within the $k^{\text {th }}$ batch. In ILC, the PI coefficients in the $k^{t h}$ batch is based on the PI coefficients in the $(k-1)^{t h}$ batch and a correcting function, which is basically dependent on the gradient of closed-loop entropy tracking error of PI coefficients in the $(k-1)^{t h}$ batch.

The $k^{t h}$ component of $\frac{\partial H(e)}{\partial K_{P}}$ and $\frac{\partial H(e)}{\partial K_{I}}$ can be approximated as follows:

$$
\begin{aligned}
\frac{\partial H(e)}{\partial K_{P}} & =\frac{H^{k-1}(e)-H^{k-2}(e)}{\Delta K_{P}} \\
\frac{\partial H(e)}{\partial K_{I}} & =\frac{H^{k-1}(e)-H^{k-2}(e)}{\Delta K_{I}}
\end{aligned}
$$

where

$$
\begin{array}{r}
\Delta K_{P}=K_{P}(k-1)-K_{P}(k-2) \\
\Delta K_{I}=K_{I}(k-1)-K_{I}(k-2)
\end{array}
$$

The statement of the convergence in this section may raise some issues about the learning parameters, type of convergence that need to be addressed more clearly. Therfore, it should be noted that the proposed convergence analysis is in introductory stage of development and future efforts need to address constraints on learning rates precisely. As it will be seen, the so called convergence laws in this work determine the constraints based on which the ILC-based cost function is decreasing along with the batches. For simplicity, we only discuss the suffcient convergence conditions to guarantee the convergence of the above proposed ILC algorithm. The key issue is that the learning rate of PI coefficients should be decreased batch-by-batch. In other words, the entropy of closed-loop tracking error should be decreased batch-bybatch. This would be equivalent to

$$
H^{k}(e(i))<H^{k-1}(e(i))
$$

Since $\alpha>0$, then inequality (19) can be re-written as

$$
\log \left(V_{R a}^{k-1}(e(i))\right)<\log \left(V_{R a}^{k}(e(i))\right)
$$

which means that

$$
\log \left(\frac{V_{R a}^{k-1}(e(i))}{V_{R a}^{k}(e(i))}\right)<0
$$

which is equivalent to the following

$$
\frac{V_{R a}^{k-1}(e(i))}{V_{R a}^{k}(e(i))}<1
$$

Since the IP is non-negative, inequality (20) would mean

$$
\Delta V_{R a, k}=V_{R a}^{k}(e(i))-V_{R a}^{k-1}(e(i))>0
$$

By using (11), the following approximation can be made.

$$
\begin{aligned}
& \Delta V_{R a, k} \approx \Delta\left[\frac{1}{N^{\alpha}} \sum_{i=1}^{N}\left[\sum_{j=1}^{N} K_{\sigma}\left(e_{i}-e_{j}\right)\right]^{\alpha}\right] \\
&=\frac{1}{N^{\alpha}} \sum_{i=1}^{N}\left\{\left[\sum_{j=1}^{N} K_{\sigma}\left(e_{k}(i)-e_{k}(j)\right)\right]^{\alpha-1} \times\right. \\
& {\left.\left[\sum_{j=1}^{N} \grave{K}_{\sigma}\left(e_{k}(i)-e_{k}(j)\right) \Delta\left(e_{k}(i)-e_{k}(j)\right)\right]\right\} }
\end{aligned}
$$


As such, when PI controller is running, the learning parameters $\lambda_{P}$ and $\lambda_{I}$ must be chosen so that the (19) to (24) together with (18) are satisfied. The algorithm can be summarized as follows:

Step 1: Collect error sample from interval $T^{k-1}$

Step 2: At the beginning of interval $T^{k}$, calculate the rate of changes of the closed-loop tracking error entropy as shown in (17).

Step3: Update the PI coefficients using (16)

Step4: Calculate the control signal using (12), and apply it to the system, and return to step 1.

This process will repeat untill the end of of control horizon is reached.

\section{Closed Loop Stability}

In this section, the stability of the closed-loop system given by (4) and (12) will be considered. Corresponding to (4) and (12), the closed-loop system equation within the $k^{\text {th }}$ batch can be written as

$$
\left.M_{k}(i+1)=\bar{A}_{k} M_{k}(i)+\bar{B}_{k} \cdot r+\bar{F} g\left(M_{k}(i)\right)\right)
$$

where

$$
M_{k}(i)=\left[\begin{array}{c}
\left.e_{k}(i)\right) \\
\xi(i)
\end{array}\right], \bar{A}_{k}=\left[\begin{array}{cc}
A-B K_{P} & -B K_{I} \\
T_{s} I & I
\end{array}\right]
$$

and

$$
\bar{B}_{k}=\left[\begin{array}{c}
I-A \\
0
\end{array}\right], \bar{F}=\left[\begin{array}{ll}
I & 0 \\
0 & 0
\end{array}\right], g\left(M_{k}(i)\right)=\left[\begin{array}{c}
g(x(i)) \\
0
\end{array}\right]
$$

To prove the stability of the closed loop system, let us consider the following Lyapunov candidate

$$
\begin{aligned}
V\left(M_{k}(i), i\right) & =M_{k}^{T}(i) P_{k}^{-1} M_{k}(i) \\
& +\lambda^{2} \sum_{i=1}^{k-1}\left[\left\|\bar{U} M_{k}(i)\right\|^{2}-\| g\left(M_{k}(i) \|^{2}\right]\right]
\end{aligned}
$$

Differentiating equation (28) over the time gives

$$
\begin{aligned}
\Delta V(i) & =V\left(M_{k}(i+1), i+1\right)-V\left(M_{k}(i), i\right) \\
& =\bar{S}_{k}^{T}(i) N_{k} \bar{S}_{k}(i)+2 \bar{S}_{k}(i) \overline{A_{k}^{T}} P_{k}^{-1} \bar{B}_{k} r \\
& +2 g^{T}\left(M_{k}(i) \overline{F_{k}^{T}} P_{k}^{-1} \overline{B_{k}} r\right. \\
& +r^{T} \bar{B}_{k}^{T} P_{k}^{-1} \bar{B}_{k} r<0
\end{aligned}
$$

where

$$
N_{k}=\left[\begin{array}{cc}
\overline{A_{k}^{T}} P_{k}^{-1} \overline{A_{k}}-P_{k}^{-1}+\lambda^{2} \overline{U^{T}} \bar{U} & \overline{A_{k}^{T}} P_{k}^{-1} \bar{F} \\
\bar{F}^{T} P_{k}^{-T} \overline{A_{k}} & \overline{F^{T} P_{k}^{-1} \bar{F}-\lambda^{2} I}
\end{array}\right]
$$

Using the well known Schur complement formula, (30) can be further reduced to

$$
N_{1, k}=\left[\begin{array}{ccc}
-P_{k}^{-1}+\lambda^{2} \bar{U}^{T} \bar{U} & 0 & \overline{A_{k}^{T}} \\
0 & -\lambda^{2} I & \bar{F}^{T} \\
\overline{A_{k}} & \bar{F} & -P_{k}
\end{array}\right]<0
$$

By pre-multiplying $N_{1, k}$ by $\operatorname{diag}\left(P_{k}^{T}, I, I\right)$ and post multiplying it by $\operatorname{diag}\left(P_{k}, I, I\right)$ and also applying Schur complement formula, the necessary condition for stability will be as follows

$$
N_{2, k}=\left[\begin{array}{cccc}
-P_{k} & 0 & P_{k}^{T} \overline{A_{k}^{T}} & \lambda \cdot P_{k} \overline{U_{k}^{T}} \\
0 & -\lambda^{2} I & F^{T} & 0 \\
\bar{A}_{k} P_{k} & \bar{F} & -P_{k} & 0 \\
\lambda \bar{U}_{k} P_{k} & 0 & 0 & -I
\end{array}\right]<0
$$

If (32) holds, a positive scalar $\delta$ exists so that $N_{k} \leq-\delta . I$. Along with (25) it can be verified that

$$
\begin{aligned}
\Delta V(i) & \leq-\delta\left\|\bar{S}_{k}\right\|^{2}+2\left\|\overline{A_{k}^{T}} P_{k}^{-1} \bar{B}_{k} r\right\| \cdot\left\|\bar{S}_{k}\right\|^{2} \\
& +2 g^{T}\left(M_{k}(i)\right) \overline{F_{k}^{T}} P_{k}^{-1} \bar{B}_{k} r \\
& +r^{T} \overline{B_{k}^{T}} P_{k}^{-1} \bar{B}_{k} r
\end{aligned}
$$

It is obvious that the right-hand side of inequality (33) is a second degree polynomial with respect to $\bar{S}_{k}(i)$. It can be shown that $\Delta V(i) \leq 0$ holds if

$$
\begin{aligned}
\bar{S}_{k}(i) & \geq \delta^{-1}\left(\left\|\overline{A_{k}^{T}} P_{k}^{-1} \overline{B_{k}} r\right\|\right. \\
& \left.+\sqrt{\left\|\overline{A_{k}^{T}} P_{k}^{-1} \overline{B_{k}} r\right\|^{2}+\delta\left(c_{1}+c_{2}\right)}\right)
\end{aligned}
$$

where

$c_{1}=2 g^{T}\left(M_{k}(i)\right) \overline{F_{k}^{T}} P_{k}^{-1} \overline{B_{k}^{T}} r$ and $c_{2}=r^{T} \overline{B_{k}^{T}} P_{k}^{-1} \overline{B_{k}} r$

This means that the stability of the closed-loop system can be checked.

\section{Simulation Results}

Using the Lagrange Euler formulation, the dynamic equation of a n-joint of an ideal rigid robot arm can be expressed with the equation of motion given by [22],

$$
M(q) \ddot{q}+c(q, \dot{q}) \dot{q}+g(q)=u
$$

where $q \in R^{n}$ is the joint angular position vector of the robot manipulator; $u \in R^{n}$ is the applied joint torques; $M(q) \in$ $R^{m \times n}$ is the inertia matrix; $c(q, \dot{q}) \in R^{m \times n}$ is the effect of Coriolis and centrifugal forces; $g(q) \in R^{n}$ is the gravitational torques. In this work, a two-link robot manipulator as shown in Fig. 2 is considered. The parameter matrices are as follows [23]:

$$
\begin{gathered}
M(q)=\left[\begin{array}{cc}
\theta_{1}+\theta_{2}+2 \theta_{3} \cos \left(q_{2}\right) & \theta_{2}+2 \theta_{3} \cos \left(q_{2}\right) \\
\theta_{2}+2 \theta_{3} \cos \left(q_{2}\right) & \theta_{2}
\end{array}\right] \\
c(q, \dot{q})=\left[\begin{array}{cc}
-\theta_{3} \sin \left(q_{2}\right) \dot{q}_{2} & -\theta_{3} \sin \left(q_{2}\right)\left(\dot{q}_{1}+\dot{q}_{2}\right) \\
\theta_{3} \sin \left(q_{2}\right) \dot{q}_{1} & 0
\end{array}\right], \\
g(q)=\left[\begin{array}{c}
g\left(\theta_{4}+\theta_{5}\right) \cos \left(q_{1}\right)+g \theta_{6} \cos \left(q_{1}+q_{2}\right) \\
g \theta_{6} \cos \left(q_{1}+q_{2}\right)
\end{array}\right],
\end{gathered}
$$

where $g$ is the gravitational acceleration and

$$
\begin{gathered}
\theta_{1}=m_{1} l_{c 1}^{2}+m_{2} l_{1}^{2}+I_{1} \\
\theta_{2}=m_{2} l_{c 2}^{2}+I_{2} \\
\theta_{3}=m_{2} l_{1} l_{c 2} \\
\theta_{4}=m_{1} l_{c 1}
\end{gathered}
$$




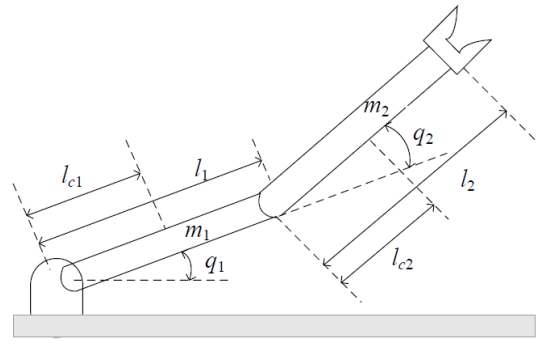

Fig. 2. Two-link robot manipulator.

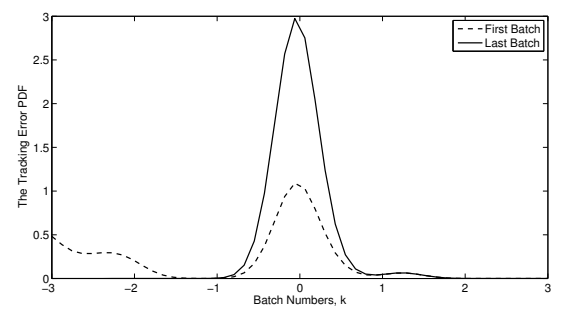

Fig. 3. The erroe PDF for the first and last batch

$$
\begin{gathered}
\theta_{5}=m_{1} l_{1} \\
\theta_{6}=m_{2} l_{c 2}
\end{gathered}
$$

The numerical values for the two-link robot are presented in Table I. The desired trajectories are:

$$
q_{d}=\left[\begin{array}{l}
q_{d 1} \\
q_{d 2}
\end{array}\right]=\left[\begin{array}{l}
3.14(1-\exp (-0.5 t)) \\
3.14(1-\exp (-0.5 t))
\end{array}\right]
$$

TABLE I

THE PARAMETER VALUES OF TWO-LINK ROBOT

\begin{tabular}{|c|c|c|c|c|}
\hline $\mathrm{m}_{1}=m_{2}$ & $1_{c 1}=l_{c 2}$ & $1_{1}=l_{2}$ & $\mathrm{I}_{1}=I_{2}$ & $\mathrm{~g}$ \\
\hline $10 K g$ & $0.5 m$ & $1 m$ & $1 K g \cdot m^{2}$ & $9.81 K g / s^{2}$ \\
\hline
\end{tabular}

The robotic control systems under communication environment are stochastic in nature because of the random time delays caused by the communications on the employed networks. However, for the simulation in this work, it is assumed that the system (35) is stochastic in the sense that it is corrupted with the process noise $d$ which follows non-Gaussian distribution. Equation (35) can be written as

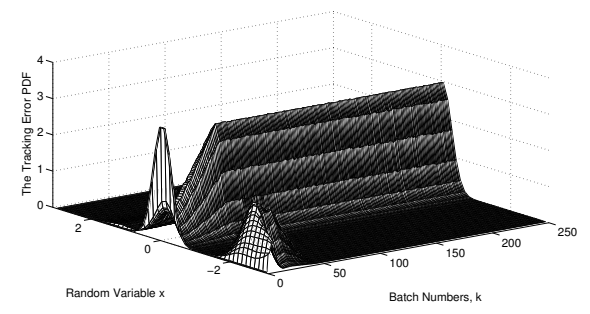

Fig. 4. The 3D mesh of the tracking error through ILC

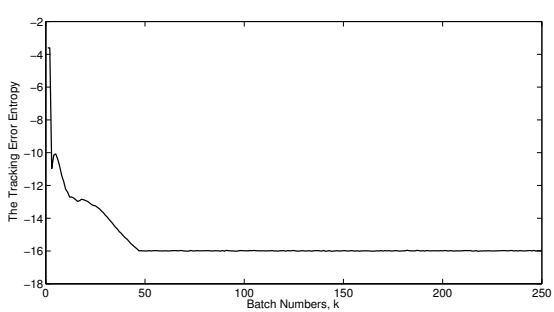

Fig. 5. The error entropy along with the batches

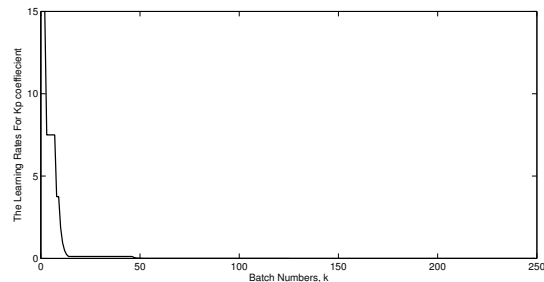

Fig. 6. $\lambda_{P}$ converge of two-link robot

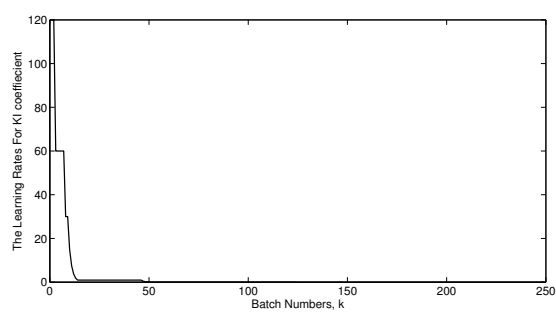

Fig. 7. $\lambda_{I}$ converge of two-link robot

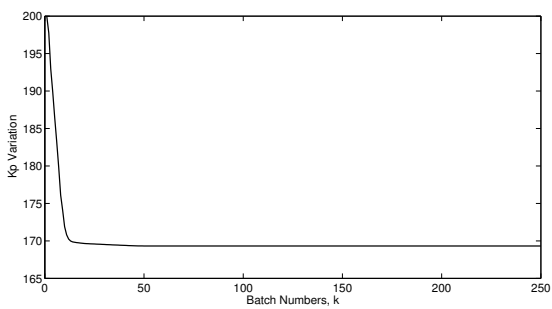

Fig. 8. $K_{P}$ variation of two-link robot

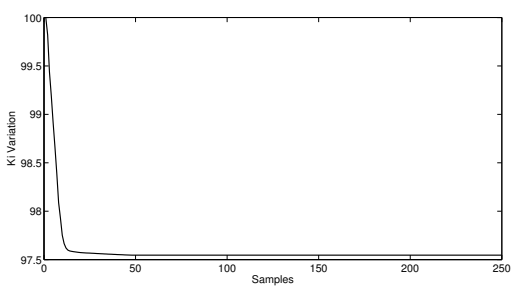

Fig. 9. $K_{I}$ variation of two-link robot 


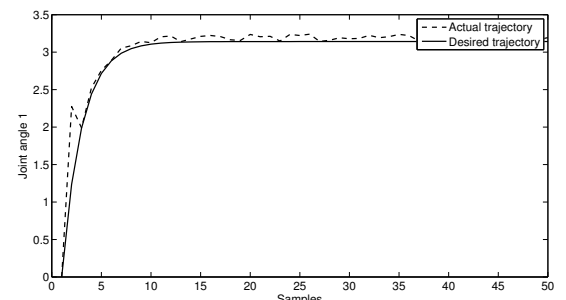

Fig. 10. The response of actual and desired path of link 1

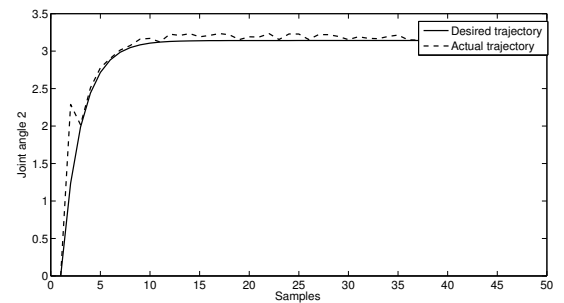

Fig. 11. The response of actual and desired path of link 2

follows:

$$
M(q) \ddot{q}+c(q, \dot{q}) \dot{q}+g(q)+d=u
$$

Assuming the following initial value $[1 ; 1 ; 0 ; 0]$. Fig. 3 shows that the proposed algorithm works as expected by comparing the shape of error PDF in the first batch and the last iteration. Moreover, the 3-D mesh of the PDF of the closed loop tracking error further confirms that the closed loop tracking error tends to be a Gaussian-like shape, as shown in Fig. 4. Although some small fluctuations can be seen in the entropy variations, the overall trend of the closed-loop error entropy suggests a minimum has been achieved. Fig. 5 illustrates how the closed-loop error entropy is minimised along with the batches.

By choosing the ILC learning rates $\lambda_{P}(1)=2, \lambda_{I}(1)=$ 5. Figs. 6 and 7 show the ILC learning rates converge throughout the batches. Also, the ILC-based tuning algorithm sets PI controller to $K_{P}=169.3 I$ and $K_{I}=79.5 I$ in the last batch as shown in Figs 8 and 9. From simulated results it can be seen that both $q_{1}$ and $q_{2}$ converge to the desired trajectories in Figs. 10 and 11.

\section{ACKNOWLEDGMENTS}

This work is jointly supported by the Chinese National Fundamental Research Program (No. 2009CB320604), the National Natural Science of China (No. 61074071,60534010), UK EPSRC (EP/I016643/1, EP/E050441/1-CICADA), the Funds for Creative Research Groups of China (No.60821063), and 111 Project (No. B08015).

\section{REFERENCES}

[1] K. Astrom, Introduction to Stochastic Control Theory. New York: Academic Press, 1970.

[2] B. Anderson and J. Moore, Linear Optimal Control. Englewood Cliffs, NJ: Prentice Hall, 1971.
[3] V. Solo, "Stochastic adaptive control and martingale limit theory," Automatic Control, IEEE Transactions on, vol. 35, no. 1, pp. 66-71, 1990

[4] E. Yaz, "Optimal stochastic control for performance- and stabilityrobustness," Automatic Control, IEEE Transactions on, vol. 38, no. 5, pp. 757-760, 1993.

[5] N. Filatov and H. Unbehausen, "Adaptive predictive control policy for nonlinear stochastic systems," Automatic Control, IEEE Transactions on, vol. 40, no. 11, pp. 1943-1949, 1995.

[6] M. Everdij and H. Blom, "Embedding adaptive jlqg into lq martingale control with a completely observable stochastic control matrix," $\mathrm{Au}$ tomatic Control, IEEE Transactions on, vol. 41, no. 3, pp. 424-430, 1996.

[7] Y. Xia, P. Shi, G. Liu, and D. Rees, "Sliding mode control for stochastic jump systems with time-delay," in Intelligent Control and Automation, 2006. WCICA 2006. The Sixth World Congress on, vol. 1 , pp. 354-358, 2006.

[8] H.-N. Wu and K.-Y. Cai, "Mode-independent robust stabilization for uncertain markovian jump nonlinear systems via fuzzy control," Systems, Man, and Cybernetics, Part B: Cybernetics, IEEE Transactions on, vol. 36, no. 3, pp. 509-519, 2005.

[9] W. Bo and J. Haibo, "Robust hinf control of uncertain stochastic nonlinear systems driven by noise of unknown covariance," in Control Conference, 2008. CCC 2008. 27th Chinese, pp. 779-784, 2008.

[10] L. Man, W. Zhao-jing, W. Wei, and D. Xiao-dong, "Adaptive outputfeedback control for stochastic nonlinear systems with zero dynamics," in Control and Decision Conference, 2009. CCDC '09. Chinese, pp. 1793-1800, 2009.

[11] H. Wang, Bounded dynamic Stochastic Systems: Modelling and Control. London: Springer-Verlag, 2000.

[12] H. Wang and H. Yue, "Minimum entropy control of non-gaussian dynamic stochastic systems," in Decision and Control, 2001. Proceedings of the 40th IEEE Conference on, vol. 1, pp. 382-387, 2001.

[13] H. Yue and H. Wang, "Minimum entropy control of closed-loop tracking errors for dynamic stochastic systems," Automatic Control, IEEE Transactions on, vol. 48, no. 1, pp. 118-122, 2003.

[14] C. Shannon, A mathematical theory of communication. The Bell System Technical Journal, 1948.

[15] N. Pal and S. Pal, "Entropy: a new definition and its applications," Systems, Man and Cybernetics, IEEE Transactions on, vol. 21, no. 5, pp. 1260-1270, 1991

[16] B. Silverman, Density Estimation for Statistics and Data Analysis. Chapman and Hall/CR, 1992.

[17] C. Yang and H. Wang, "Minimum entropy control approach of closed-loop tracking errors for dynamic stochastic systems using yula parameterization formula," in Control Conference, 2006. CCC 2006. Chinese, pp. 1500-1505, 2006.

[18] J. Zhang and H. Wang, "Minimum entropy control of nonlinear arma systems over a communication network," Neural Computing and Applications, vol. 17, no. 4, pp. 385-390, 2008.

[19] L. Guo and H. Wang, "Fault detection and diagnosis for general stochastic systems using b-spline expansions and nonlinear filters," IEEE Transactions on Circuits and System, vol. 52, no. 8, pp. 16441652,2005

[20] L. Guo and H. Wang, "Applying constrained nonlinear generalized pi strategy to pdf tracking control through square root b-spline models," International Journal of Control, vol. 77, no. 17, pp. 1481-1492, 2004.

[21] D. Owens and J. Htnen, "Iterative learning control- an optimization paradigm," Annual Reviews in Control, vol. 29, no. 1, pp. 57-70, 2005.

[22] M. Spong, "On the robust control of robot manipulators," Automatic Control, IEEE Transactions on, vol. 37, no. 11, pp. 1782-1786, 1992.

[23] K.-K. Shyu, P.-H. Chu, and L.-J. Shang, "Control of rigid robot manipulators via combination of adaptive sliding mode control and compensated inverse dynamics approach," Control Theory and Applications, IEE Proceedings -, vol. 143, no. 3, pp. 283-288, 1996. 